\section{Körperpsychotherapie: Die «Nahtstelle» zwischen Körper und Psyche}

\section{T. P. Ehrensperger}

In der Zeit des Wertewandels wird für einzelne und Organisationen Identität immer mehr zur Aufgabe, weil die Anforderungen der Gesellschaft von uns den vollen Einsatz unserer Ressourcen und unseres Potentials verlangen. Präsenz, verstanden als Einheit von Körper und Seele, Flexibilität und Sensibilität, gepaart mit emotionaler Intelligenz und emotionaler Kompetenz, sind notwendige Eigenschaften in einer immer komplexeren und schnelleren Welt.

Körperpsychotherapeutische Methoden haben in den letzten 20 Jahren an Boden gewonnen, nicht nur im therapeutischen Bereich, sondern auch in anderen Berufsgruppen wie Lehrer, Tänzer, Schauspieler, Manager usw. In der Medizin zeigen uns körperpsychotherapeutische Verfahren, dass ein echt ganzheitlicher Ansatz für die Praxis damit möglich wird. Neben dem Gespräch und gewohnten Kontakt mit dem Patienten gibt es auch ein umfangreiches diagnostisches und technisches Instrumentarium zum Verstehen und effizienten Behandeln von komplexen Störungen im psychosomatischen Bereich.

Mehrere internationale Studien zeigen, dass nur bei etwa 16\% aller Patienten eine primär organische Ursache gefunden wird, bei den andern oft unnötige, teuere und ineffiziente Untersuchungen und Therapien durchgeführt werden. Dieses andauernde Missverständnis trägt zu den riesigen Kostenanstiegen in unserem Gesundheitssystem bei, ohne dabei die angewandte Gesundheitsfürsorge zu verbessern. Demgegenüber haben relativ kurzdauernde psychosoziale Interventionen immer wieder einen signifikanten Effekt gezeigt, das Inanspruchnahmeverhalten zu reduzieren.

Dass psychotherapeutische Interventionen bei psychosomatischen Krankheiten hilfreich sein können, ist natürlich schon lange bekannt, ebenso bekannt ist aber, dass gerade Patienten mit Somatisierungsstörungen wenig motiviert sind für einen primär psychotherapeutischen Zugang zu ihrer Leidensgeschichte. Hier greift nun Körperpsychotherapie in die Lücke und zeigt Wege auf, wie man über einen primär körperlichen $\mathrm{Zu}$ gang direkter und schneller zur Motivation und Mitarbeit des Patienten gelangt, weil der Therapeut nicht primär nach einem psychischen Konflikt sucht, son- dern mit Hilfe seiner diagnostischen und therapeutischen Methoden direkt einen Bezug zum bestehenden Körpergeschehen und zur Symptomatik schaffen kann.

Körperpsychotherapeutische Verfahren haben sich in den letzten Jahren besonders im deutschen Sprachbereich enorm entwickelt: In der Schweiz gibt es zwölf Institute, die sich mit der Methode in Lehre und Forschung beschäftigen, eine Dachorganisation (CHEABP) gibt eine Netzwerkstruktur und kümmert sich um Solidarität, Kommunikation und Qualitätssicherung in der Methode und fördert den Austausch zwischen ihren Vertretern.

Wirksamkeit, Wirtschaftlichkeit und Zweckmässigkeit von Körperpsychotherapie zeigt eine soeben erschienene Studie von C. Ventling und U. Gerhard [1]. Es handelt sich um eine Katamnesestudie, die aufzeigt, dass auch sieben Jahre nach Abschluss der Therapie positive Resultate vorliegen und, was besonders wichtig ist, auch anhalten.

In einer Übersichtsstudie zu den «Ökonomischen Folgen von Fehlbehandlungen psychosomatischer und somatopsychischer Erkrankungen" äussert sich F. Lamprecht, Vorsteher der Abteilung für Psychosomatik und Psychotherapie an der Medizinischen Hochschule Hannover [2]. Diese Studie berechnet Kosteneinsparungen von 120 Milliarden DM pro Jahr in Deutschland, falls es gelingt, "die ineffizienten und teuren Diagnose- und Behandlungsversuche bei psychosomatischen Störungen durch geeignete ganzheitliche psychosomatische Behandlung und Betreuung zu ersetzen".

Vom 16. bis 18. November 2000 findet in Basel der "2. Schweizerische Kongress für körperbezogene Psychotherapie" statt, bei welchem auch international Bekannte ("opinion leaders") Hauptreferate halten werden, daneben finden 60 parallele Workshops und Seminare statt. Ich möchte Sie herzlich dazu einladen. Info: Kongresssekretariat, R. Birsinger, Telefon 061 30310 10, Fax 06130141 61, E-mail: tpehrens@ hin.ch, Homepage: www.hin.ch/ch-eabp.

Der Kongress steht unter folgendem Titel: «Das Sichtbare und das Verborgene in der Körperpsychotherapie». Als Hauptreferenten konnten verpflichtet werden: Wolf Büntig, Zist, München; Angela Klopstech, Internationales bioenergetisches Institut, New York; George Downing, Paris; Malcolm und Katherine Brown, USA; Professor Siegfried Frey, Duisburg; Professor Fritz Popp, Neuss; David Boadella, Leiter des Internationalen Institutes für Biosynthese, Heiden; Pamela Chubbuck, USA; Prof. Ludwig Janus, Heidelberg/D; Franz Renggli, Basel, und andere.

Das detaillierte Kongressprogramm mit Abstracts erschien Ende September und wird auf Anfrage an alle Interessenten verschickt.
Korrespondenz:

Dr. med. Thomas P. Ehrensperger

Präsident CH-EABP

Wielandplatz 2

CH-4054 Basel

\section{Literatur}

1 Ventling C, Gerhard U. Zur Wirksamkeit bioenergetischer Psychotherapien und Stabilität des Therapieresultats: Eine retrospektive Untersuchung. Psychotherapeut 2000;45:230-6.

2 Lamprecht F. Die ökonomischen Folgen von Fehlbehandlungen psychosomatischer und somatopsychischer Erkrankungen. Psychother Psychosom Med Psychol 1996;46:283-91. 